Health \& Medicine | Lijuan Yuan

\section{Human norovirus animal model essential for vaccine development}

Human noroviruses are the most common cause of acute gastroenteritis and represent an incredibly high burden on the healthcare sector. Curren due to a lack of animal mods. Hen or Virginia Polytechnic Institute and State University developed and at gnotobiotic pigs as an animal model for the human norovirus GII.4/2003 Cin-2. Determining dose-response relationships as well as the median infectious dose, by using different statistical approaches for this virus, means we are one step closer on the path to vaccine development.

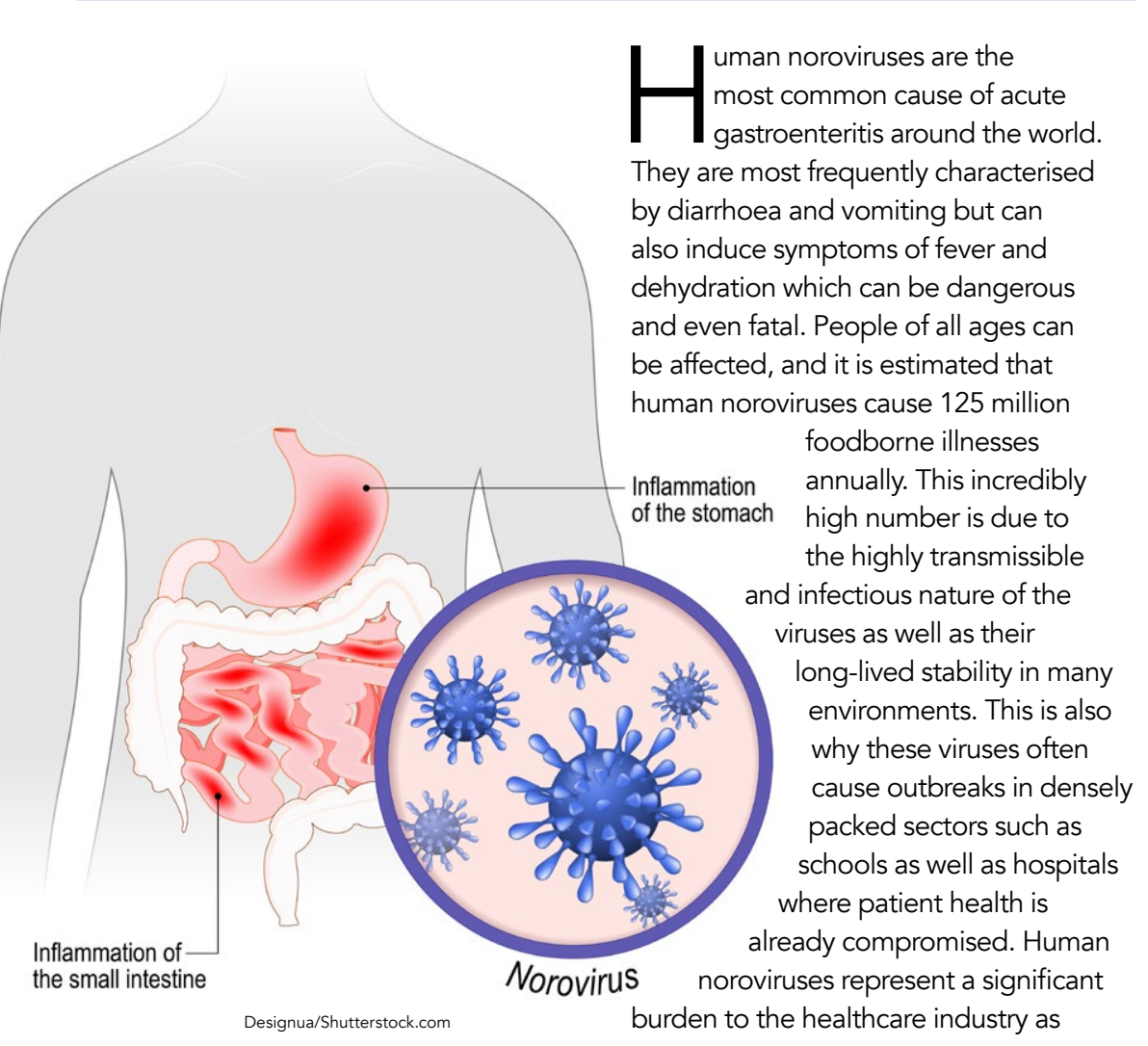

ell as having an enormous financial cost. Despite this, there are no norovirus vaccines to prevent the disease or any anti-viral medicines to treat it.

\section{THE IMPORTANCE}

OF ANIMAL MODELS

Before being accepted for human

clinical trials, antivirals, antibodies, and

vaccines need to first demonstrate their fectiveness in animal models. The

lack of an animal model for studying

his norovirus infection and disease her antiis now known that make models. Gnotobiotic pigs are raised in sterile, germ-free environments so all microbe exposures can be accounted for. This means that, if infected with an organism, it's clear that any symptoms that develop will have stemmed from this. These pigs are good candidates as animal models due to their shared similarities with humans. They have similar immune responses as well as virus-binding patterns. This means that disease progression and infection doses determined from these pigs are comparable to what would occur in humans.

key factor that can be learnt from thes is models is the median infectious the virus that mus be (or dose) of of the population to become infected the animal-model study. The median vacio methods can provide an estimate, but more contemporany mechanistic doseresponse models may prove to be more effective and accurate. These newer methods can also give an overall picture about infection at any dose level, not just $50 \%$. Once this has been established, the median infectious dose can then be used as a starting point for human pre-clinical studies or risk assessments to do with food and water safety. Since the results between animals and humans is comparable, this ensures safety for testing high levels of the virus.

HUMAN NOROVIRUS STUDIES SING GNOTOBIOTIC PIG Dr Lijuan Yuan and the team at Virginia have been carning out studies on huma noroviruses in gnotobiotic pigs for many years. They established the gnotobiotic pig model of human norovirus infection and diarrhoea for vaccine evaluation. They have also carried out experiments to test changes in infection and immunity in response to various factors. It has been discovered that probiotics provide protection against norovirus while the drug simvastatin

enhances the infection. They have also looked into the relationship between noroviruses and the human gut microbiota by

gut bacteria to these gnotobiotic pigs.

Their most recent study however, focuses

Human noroviruses represent a significant burden to global health as well as having an enormous financial cost. seven different groups with each group
being inoculated with a different dilution
or dose of the virus. Samples were then taken every day from the animals and
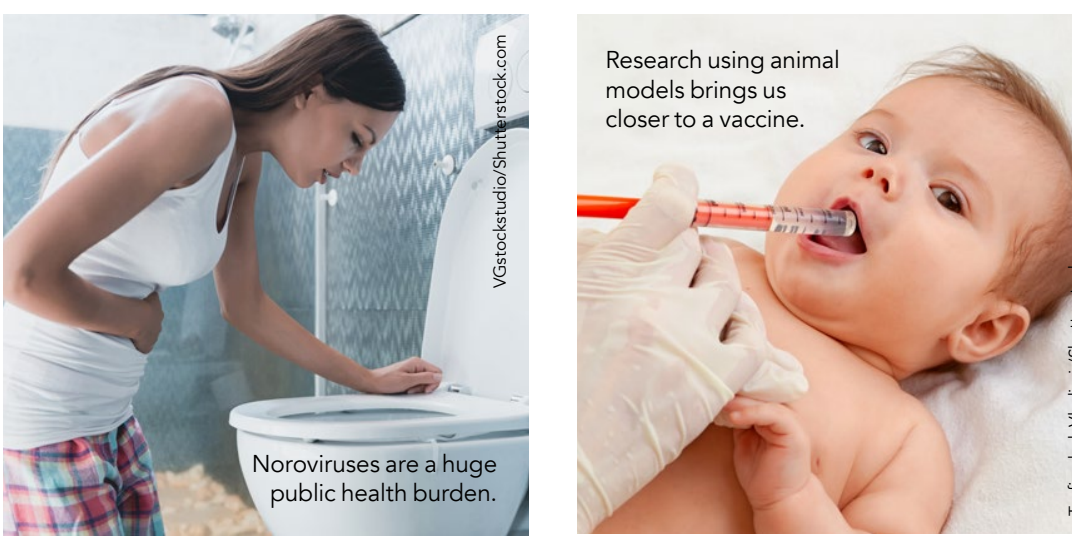

analysed for viral RNA that had been shed. Diarrhoea symptoms were also ssessed daily for seven days.

ESTABLISHING THE DOSEFrom these experiments it was

dermined, as expected, that higher$$
\text { doses of virus had }
$$

shorter incubation periods, so animals were infected more quickly. Infection persisted in animals infected with higher shed higher amounts

of virus overall in the study than the animals given lower doses. Similarly, the diarrhoea symptoms appeared earlier and for longer in the animals given a higher dose of virus. Pigs given a dose of $2 \times 10^{5}$ RNA copies of viral genomes had the highest and longest level of viral shedaing and diarrhoea symptoms. On average they were shedding viral diarto for 6.3 out of the 7 days and noculation lasting for 4 days. This makes $2 \times 10^{5}$ the optimal challenge dose for this GII.4/2003 human norovirus. This was the third-highest dose of all the groups. These results were similar to those from a previous human volunteer challenge
study, showing again that the gnotobiotic 

administered need to be equally spaced logarithmically and the number of animals has to be the same in each group tested.

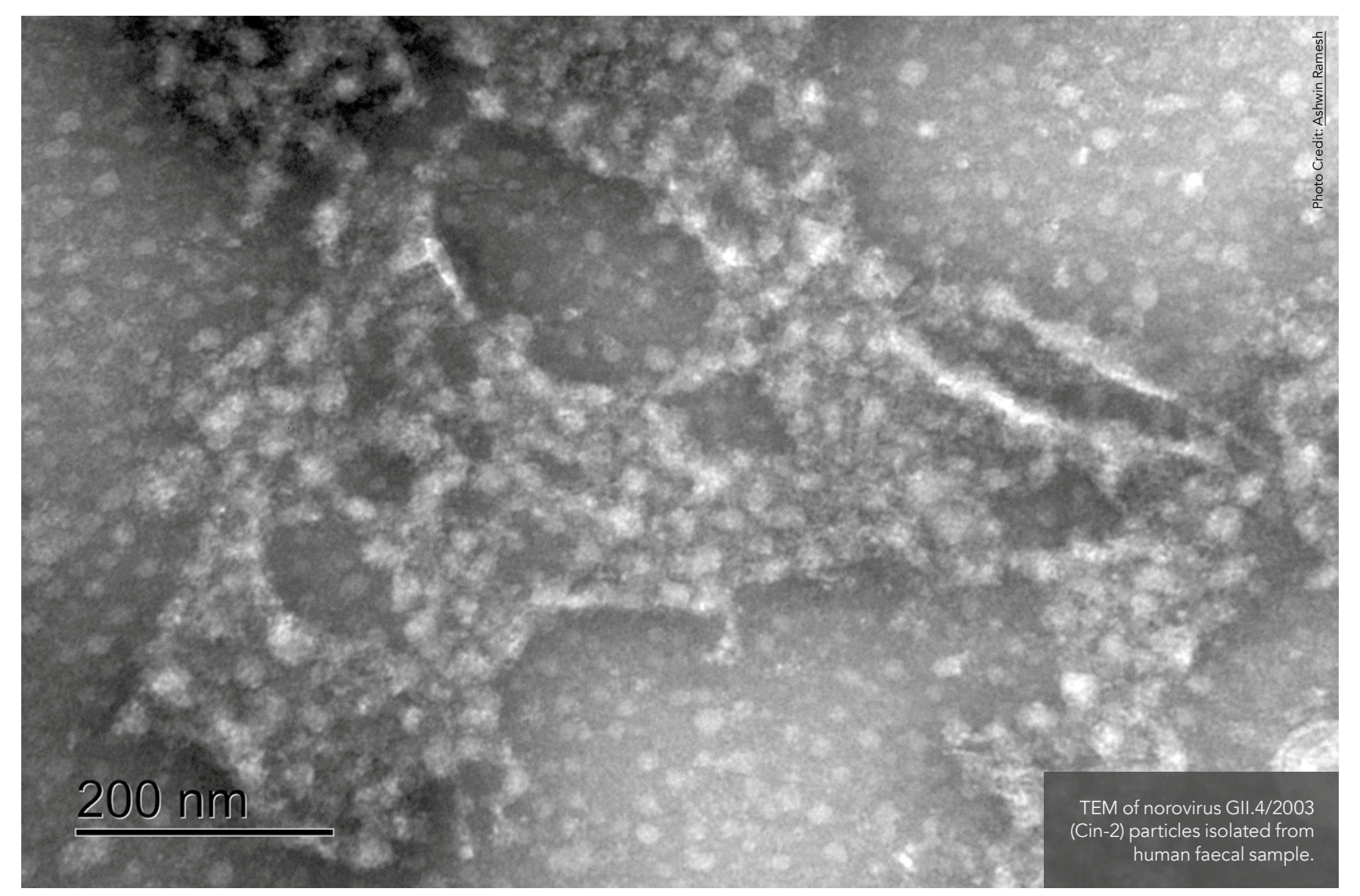

pigs are a replicable animal model in

and flexible estimations. Using all of these calculations it was determined that the median infectious dose was between $2.4 \times 10^{3}$ and $3.4 \times 10^{3}$ RNA copies of vira genomes while the median diarhoea dose was between $2.1 \times 10^{4}$ and $3.8 \times 10^{4}$ RNA copies of viral genomes. out the median infectious dose and the median diarrhoea dose. Classi methods can be useful in these experiments, but they were developed sefore more accessible computer access, so they do have some shortcomings. de It is worth noting that all these analyses were carried out with the assumption that However, ithas been seen in previous

means that the median doses determin were for aggregated particles and it viral particles would give lower media infectious and diarrhoea doses.

\section{THE PATH TO} VACCINE DEVELOPMENT These experiments have shown again hat responses to human noroviruses in the responses seen in humans.

The Spearman-Karber test, on the oth hand, has the drawback of needing a $0 \%$ and a $100 \%$ response in order to develop a dose-response curve. In some cases, this isn't possible, for example, pigs in each group shed viral particles so there was no $\%$ response. Exponential and beta-Poisson were more contemporary analyse results. These give mon used to
These experiments have shown that infection and disease caused by human noroviruses in gnotobiotic pigs resemble those seen in humans.

studies in which other human noroviruses were visualised by electron microscopy that they are often aggregated. The viral particles are assembled within survive longer. Electron microscopy was therefore carried out on the noroviruses used in these expenments and confirm membrane-bound vesicles which gives them more stability and means they can
Disease presents

same way. Analysing dose-response relationships using various methods leads to a better understanding of how human noroviruses

cause disease. Knowing the full extent of how much virus is needed to cause disease and induce symptoms means for testing novel vaccine candidates, The development of vaccines and drugs against human noroviruses is essential and reduce the but the discase, healthcare sector globally. that an optimal dose can be worked out

\section{Behind the Research (1) Dr Lijuan
Yuan \\ E: lyuan@vt.edu 7i Dr Ashwin \\ Ramesh E: akramesh@vt.edu a Parreno T: +1 5402319053 W: vetmed.vt.edu/research/labs/faculty-labs/yuan-lab.html

Research Objectives

Dr Lijuan Yuan and team investigate the use of gnotobiotic pigs as an animal model for the human norovirus GIll.4/2003 Cin-2.

\section{Detail}

Address Pathobiology, Virginia-Maryland College of Veterinary Medicine, Virginia Tech,

Blacksburg, VA 24061, USA.

Bio

Dr Lijuan Yuan is a professor of virology Ramm DrViviana Parreno, an investigator at

\section{References}

- Ramesh, AK, Parreño, V, Schmidt, PJ, et al, (2020) Contemporary Methods for Endpoint Estimation. Viruses, 12(9), 955 doi.org/10 3390/v12090955

- Lei, S, Twitchell, EL, Ramesh, AK, et al, (2019) Enhanced

Gll.4 human norovirus infection in gnotobiotic pigs transplanted with a human gut microbiota. J Gen Viro, 100(11), 1530-1540. doi.org/10.1099/jgv.0.001336 - Lei, S, Ramesh, A, Twitchell, E, et al, (2016) High Protective Efficacy of Probiotics and Rice Bran against Human Norovirus Infection and Diarrhea in Gnotobiotic Pigs. Frontiers in Microbiology, 7, 1699. doi.org/10.3389/ fmicb.2016.01699

Bui,, , Kocher, J, Li, Y, et al, (2013) Median infectious dose of human norovirus $G$. 4 in gnotobiotic pigs is decreased 94, 2005-2016. doi.org/10.1099/vir.0.054080-0 metrics. Currently, she is a visiting research scientist in Yuan Lab.

The projectwas funded by a human ovirus vaccine evaluation contract ( Pun, July 2017-December 2018). Evaluation of the $50 \%$ fectious Dose of Human Norovirus

NTA-CONICET, Argentina, analysed

\section{Collaborators}

XI Jiang from Cincinnati Children's Hospital Medical Center, Cincinnati, OH, USA for providing the HuNoV Gll.4 inoculum pool. Philip J Schmidt and Monica B Emelko from Department of Civi Environmental Engineering, University of Waterloo, Waterloo, statistical analyses.

\section{Personal Response}

How close do you think we are to the successfu development of a human norovirus vaccine?

II We are working with collaborators in efforts to speed up the development of human norovirus vaccines. A quadrivalent virus-like particle vaccine developed by Anhui Zhifei Longcom Biologic and evaluated in our pig Ad5 vector is underphase ib clical trial (NCT04854746). There are many other ongoing efforts. A successful vaccine
is possible within the next 5 years.

$\sqrt{7}$ VIRGINIA model is currently under a phase I/la human clinical tria (NCT04563533). An oral norovirus GI. I vaccine based on 